\title{
Chronic low back pain: Can Yoga as an integrative approach be the best way forward?
}

\author{
Spoorthi Poojaria, Babita Ghaib*, Kashinath Metric, Sheetal Jindal Guptad, Prashant Verma ${ }^{\mathrm{e}}$ \\ aS-VYASA Yoga University, Bengaluru, Karnataka, India \\ ${ }^{b}$ Department of Anaesthesiology and Intensive Care, PGIMER, Chandigarh, India \\ 'Central University of Rajasthan, Bandar Sindri, Ajmer, Rajasthan, India \\ dS-VYASA Yoga University, Bengaluru, Karnataka, India \\ eNeuroscience Research Lab, PGIMER, Chandigarh, India
}

\section{KEY WORDS}

Chronic low back pain

Yoga

IAYT

\begin{abstract}
Chronic Low Back pain (CLBP) is common public health problem, and globally one of the leading cause of disability. Several research studies advocate Yoga may add on as an effective therapy for CLBP patients. The present case study is an attempt to elucidate the outcome of Integrated Approach of Yoga Therapy (IAYT) intervention (which included loosening practices, asanas with breathing, relaxation, pranayama and meditation techniques) as an adjunct and integrative therapy to reduce pain symptoms, pain-associated disability and their overall quality of life in CLBP adult patients. The manuscript illustrates the two CLBP patients, refractory to conservative management, visiting pain clinic at Post Graduate Institute of Medical Education and Research (PGIMER), Chandigarh, India. Both the patients received a series of 60 minutes IAYT class conducted for a span of 15 days along with the institutional usual care regime as their treatment protocol. Patients were evaluated at baseline, 1 month and 3 month using various assessment tools (for pain intensity, disability, neuropathic pain component, quality of life, pain experiences, mental health, fear of movements and overall clinical benefit). Both the patients showed steady and gradual progression in reducing their pain intensity and its associated disability, quality of life and mental health. The patients narrated their experience and their ability to manage bio-psycho-socio aspects associated with CLBP. They attributed their positive changes to IAYT protocol.
\end{abstract}

doi: $10.38205 /$ imcr.010106
Babita Ghai, MD

Department of Anaesthesiology and Intensive Care, PGIMER

Chandigarh, India.

Contact no: +91-7087009533

E-mail: ghaibabita1@gmail.com

\section{Introduction}

Chronic Low back pain (CLBP), is a common public health problem and a leading cause of disability worldwide $(1,2)$. Globally, low back pain has prevailed to be one of the top four leading causes of Years lived with Disability (YLDs), as calculated by the Global Burden of Disease 2017 study (3). Also alarming is the incidence of low back pain (LBP) in India, as back and neck pain is ranked second leading cause of YLDs after iron deficiency anaemia with nearly 60 percent of individuals suffering from it at some point during their lives $(3,4)$. CLBP impacts all aspects of life including biological, psychological, social, functional and financial. Various treatment options are available for CLBP management. However, there is insufficient or conflicting evidence about these modalities (5). As these modalities mainly focus on biomedical aspect of pain they usually miss the mind-body aspect. Pharmacological methods seem inadequate as a sole treatment to CLBP (5). Research studies showcase that Integrated Approach of Yoga Therapy (IAYT) can be a potential add-on and synergistic culmination for an effective management in CLBP (6-8).

Its approach and possibilities are very similar to Mindfulness Based Stress Reduction (MBSR) technique, which has been undertaken for back pain and other conditions by more than 18,000 Americans $(9,10)$. The current cases illustrate the positive outcome of IAYT intervention to reduce pain symptoms and pain-associated disability in CLBP adult patients.

\section{Case presentation}

\section{Recruitment of patient}

Patients with a history of low back pain with or without radicular pain for more than 3 months, who were refractory to previous conservative management, were recruited at the pain clinic Out-Patient Department of Post Graduate Institute of Medical Education and Research (PGIMER), Chandigarh run by the Department of Anaesthesiology and Intensive care. The initial evaluation was based on the usual practice of physician assessment based on symptoms and radio-diagnostic methods. Patients written consent were obtained prior to their enrolment for IAYT sessions.

\section{IAYT (Integrated Approach of Yoga Therapy) Protocol}

Both the patients were given IAYT as an adjunct treatment along with the Institutional Conventional regime (back 
care postural advice, hot water application and medication). A series of 60 minutes IAYT class was conducted for a span of 15 days. The Intervention was supplemented by IAYT handouts and audio for the practice of Yoga at home in the following months. Outcomes were evaluated in Pain clinic by keeping the follow up of these two patients along with other patients visiting the Pain clinic. Physician evaluating the outcome was blinded to the participation of these two patients in the IAYT sessions, hence masking the investigators regarding the treatment assignments. Assessment was done at baseline, 1 month and 3 month.
IAYT (Table 1) is a treatment protocol supported by scientific studies, blend with ancient wisdom; based on the basic principle of Panchkoshas; that is five layers of the existence to human beings hypothesized in traditional literature (6-8). The protocol involves practices at the following levels;

- Body level (Annamaya Kosa): Loosening practices and Yoga Asanas

- Subtle energy level (Pranamaya Kosa): Breathing practices and Pranayama

- Mind level (Manomaya Kosa): Meditation and Relaxation techniques.

Table 1: Integrated Approach of Yoga Therapy (IAYT) Module for Chronic Low Back Pain

\begin{tabular}{|c|l|}
\hline S. NO. & IAYT Module for Chronic Low Back Pain Patients \\
\hline 1. & Opening Prayer (To align the body, mind and spirit and enhance the benefits of Practice) \\
\hline 2. & Loosening Practices (Increases Microcirculation and preps the body) \\
\hline 3. & Specific Supine Back Practices (Asanas with Breathing) \\
\hline 4. & Quick Relaxation Technique \\
\hline 5. & Specific Prone Back Practices (Asanas with Breathing) \\
\hline 6. & Relaxation in Makarasana (Crocodile Pose) \\
\hline 7. & Standing asanas (with Breathing) \\
\hline 8. & Deep Relaxation (with Folded Legs) \\
\hline 9. & Pranayama \\
\hline 10. & Meditation \\
\hline 11. & Closing Prayer (Prayer for happiness, health and peace for all.) \\
\hline 12. & Group Discussion \& Feedback \\
\hline TOTAL 60 minutes Practice \\
$\quad+10$ minutes (Discussion) \\
\hline
\end{tabular}

\section{Diagnosis}

The assessment of patients was based on comprehensive clinical evaluation and assessing their symptoms, the degree of functional limitation and psychological wellbeing. Radio-diagnostic methods involved MRI scan of lumbosacral spine. The general parameter check up on their body weight, height, blood pressure to access general health status was done. Their Vitamin D 3 level check-up was also a part of the evaluation.

\section{Clinical Findings}

Case 1 is a 38 year old female patient with 4 years clinical history of low back pain. Her pain intensity was 7-9/10 on verbal numerical rating scale (VNRS). She complained of radicular pain in both the legs till great toe, along with pain on both posterior and anterior aspect of the right thigh and buttocks. MRI investigations of this patient revealed diffuse disc bulges at L3-4, L4-5 and L5-S1 with early disco-generative changes. (refer to supplementary data). There was aggravated pain at night, along with sleep disturbances with the patient unable to sleep on the affected right side. Pain was aggravated by standing and prevented her from standing for more than 15 minutes at a stretch. In past, she had received an epidural steroid injection (ESI) twice within the span of 3 months around 4 years back (in year 2015), which provided her 70\% pain relief only for span of two weeks after each ESI. Pain had intermittent increase over the following years with current average VNRS of 7-9/10. She was receiving usual care from PGIMER, Chandigarh for last 4 years and was compliant with her conventional treatment of regular back physiotherapy exercises and medications. Despite this the pain and disability did not show any improvement. Impact on work productivity and performance of daily usual activities was also stated, with the need for intermittent rest throughout the day.

Another Case report 2 is of a 38 year old male patient presented with a 6 years history of low back pain. The pain was radiating to the whole of left lower limb and calf region, extended to stiffness in both legs with left leg movement difficulties, especially while walking and keeping several postural positions. The back pain was exacerbated by movements with numbness and tingling sensation in the left sole while walking. 
Table 2: Patient 1; 38/Female - Assessment for the primary and secondary outcome measures

\begin{tabular}{|c|c|c|c|c|}
\hline Assessments & Tools & Baseline & 1 month & 3 month \\
\hline Pain Intensity Measure & VNRS (Verbal Numeric rating scale) & 8.5 & 3 & 1 \\
\hline Pain Disability & $\begin{array}{l}\text { MODI (Modified Oswestry Disability } \\
\text { Index) }\end{array}$ & $58 \%$ & $24 \%$ & $10 \%$ \\
\hline \multirow[t]{2}{*}{ Neuropathic Pain Measure } & Pain Detect Questionnaire & 12 & 3 & 0 \\
\hline & Douleur Neuropathique 4 (DN4) & 4 & 0 & 0 \\
\hline Quality of Life & EuroQol (EQ-5D- 5L) & $28 \%$ & $68 \%$ & $80 \%$ \\
\hline Pain Experiences & Pain Catastrophizing Scale (PCS) & 44 & 6 & 0 \\
\hline \multirow[t]{2}{*}{ Mental Health } & \multirow{2}{*}{$\begin{array}{l}\text { Hospital Anxiety and Depression } \\
\text { Scale (HADS) }\end{array}$} & $A-13$ & $A-2$ & $A-2$ \\
\hline & & $D-9$ & $D-2$ & $\mathrm{D}-2$ \\
\hline Fear of Movement & Tampa Scale of Kinesiophobia (TSK) & 39 & 28 & 27 \\
\hline \multirow[t]{2}{*}{$\begin{array}{l}\text { Clinical Benefit from } \\
\text { The treatment }\end{array}$} & $\begin{array}{l}\text { Patient Global Impression of Change } \\
\text { Scale (PGICS) }\end{array}$ & - & 2 (Much improved) & $\begin{array}{l}1 \text { (Very much } \\
\text { improved) }\end{array}$ \\
\hline & Medication-Drug Therapy and doses & Dual - $110 \mathrm{mg}$ & Dual - 85 mg & Mono - 5 mg \\
\hline
\end{tabular}

Table 3: Patient 2; 38/Male - Assessment for the primary and secondary outcome measures

\begin{tabular}{|c|c|c|c|c|}
\hline Assessments & Tools & Baseline & 1 month & 3 month \\
\hline Pain Intensity Measure & VNRS (Verbal Numeric rating scale) & 10 & 4 & 1.5 \\
\hline Pain Disability & $\begin{array}{l}\text { MODI (Modified Oswestry Disability } \\
\text { Index) }\end{array}$ & $54 \%$ & $38 \%$ & $26 \%$ \\
\hline \multirow[t]{2}{*}{ Neuropathic Pain Measure } & Pain Detect Questionnaire & 13 & 5 & 2 \\
\hline & Douleur Neuropathique 4 (DN4) & 5 & 1 & 1 \\
\hline Quality of Life & EuroQol (EQ-5D- 5L) & $32 \%$ & $56 \%$ & $76 \%$ \\
\hline Pain Experiences & Pain Catastrophizing Scale (PCS) & 44 & 12 & 6 \\
\hline \multirow[t]{2}{*}{ Mental Health } & \multirow{2}{*}{$\begin{array}{l}\text { Hospital Anxiety and Depression } \\
\text { Scale (HADS) }\end{array}$} & A - 18 & $A-7$ & $A-4$ \\
\hline & & $\mathrm{D}-18$ & $\mathrm{D}-10$ & $\mathrm{D}-4$ \\
\hline Fear of Movement & Tampa Scale of Kinesiophobia (TSK) & 41 & 36 & 31 \\
\hline \multirow[t]{2}{*}{$\begin{array}{l}\text { Clinical Benefit from } \\
\text { the Treatment }\end{array}$} & $\begin{array}{l}\text { Patient Global Impression of Change } \\
\text { Scale (PGICS) }\end{array}$ & - & $\begin{array}{l}2 \text { (Much } \\
\text { improved) }\end{array}$ & $\begin{array}{l}1 \text { (Very much } \\
\text { improved) }\end{array}$ \\
\hline & Medication-Drug Therapy and doses & Dual - $160 \mathrm{mg}$ & Mono - 75 mg & Off Medication \\
\hline
\end{tabular}

The main precipitating factors were prolonged standing, walking and sitting. MRI reported revealed disc bulge at multiple levels of L2-L3, L4-L5 1V disc level with severe posterocentral disc protrusion more so in the left side (refer to supplementary data). During the consultation, the patient appeared to be stressed out and his gait was not balanced. He admitted that the conservative treatment with analgesia and physiotherapy gave him no relief despite him being complaint to treatment. Also his episodes of acute pain have become increasingly severe over the years and were starting to interfere with his overall quality of life, so much so that, it had started affecting his mental health and his behaviour with his family. His family claimed that he had become increasingly irritable and would avoid communication with them. He had been absent from work for each episode of back pain and since the past 5 months, the patient had stopped going to work completely. His average VNRS for pain reported was $10 / 10$ for most of the day.

Both the patients were given IAYT as an adjunct treatment along with the Institutional Conventional regimen for 2 weeks session ( 6 sessions/week) under guidance by qualified Yoga trainers from SVYASA, Bengaluru. Subsequent to this, they were provided with audio tapes and pamphlets of IAYT and were encouraged to do IAYT at least 5 days/week and were followed up at 1 month and 3 months. Both patients demonstrated remarkably substantial improvement over the span of 3 months as reported by Patient Global Impression of Change Scale (PGICS).

When compared to the baseline assessments in both the cases, a gradual decrease in the intensity of low back pain, associated disability and neuropathic component was noted. The positive response of IAYT led to reduction in 
pain medications for both patients over the 3 months duration. In fact case 2 needed discontinuation of medication at 3 months. Both the patients reported that their experience with IAYT helped them to develop better awareness of their own body and improved control over their mind. All of these leading to a significant improvement in the overall quality of life, physical well-being and mental health. These assessments were noted using various assessment tools provided in tables at three time points (Table $2 \&$ Table 3). Case 2 patient managed to resume his work eventually within the span of 1 month IAYT intervention.

\section{Discussion}

CLBP impacts all aspects of life including biological, psychological, social, functional and financial and requires a biopsychosocial approach for its management. In the absence of potential definitive treatment for CLBP, there is reduced emphasis on non-pharmacological care and paradigm shift (5).

Evidence suggests stretching exercises or in combination with patient education are helpful in managing CLBP (11-13). However, these interventions mainly take care of biological aspect of pain and usually miss the cognitive mindbody aspect. Therefore, even after the patients' compliance with their physiotherapy and medications treatment, they found no relief. The complex nature of the CLBP demands shifting away from the biomedical model to a multidimensional biopsychosocial model for management. It is noteworthy that with IAYT even bio psycho and social aspect also witnessed a huge improvement in both the patients. The percentage change in pain associated disability was remarkable. In case 1 where the patient was unable to walk even for $1 / 4$ mile, increased it to 1 mile $(1.6 \mathrm{~km})$. She incorporated long evening walks in her daily schedule which also helped her reduce weight. Her time span of standing and sitting saw a shift as well, due to which she did not need to take constant breaks in between her household work.

The case 2 patient showed improvement in his duration of walking, standing, sitting as well. Earlier, while he was unable to stand or walk without support for long duration, but now he manages to go for long early morning walks with in between short breaks. Significant improvement in his overall posture was also noted. He was able to improvise on his daily routine with early rising and Yoga practice, whereas earlier his family expressed their concern of him waking up late after $11 \mathrm{am}$. With the aspect of pain taken care, in both the patients, they constantly mentioned regarding their new found self-confidence and assurance to continue with the practice.

By adopting IAYT as a daily practice, both the patients were able reduce their pain intensity substantially over the period of 3 months. Even the neuropathic component associated with CLBP was reduced to almost zero in case 1 and negligible in the case 2 .

IAYT has been studied by few researchers and has been reported to act as a adjunct for an effective holistic management in Chronic Low Back Pain in Indian population (6-8). In CLBP patients, the anticipation of pain due to physical movement is often accompanied by anxiety and depression. These further exacerbate the mental state described above. A study infers that the rate of major depression is $20 \%$ more with CLBP group, compared to $6 \%$ for pain- free individuals $(14,15)$. It was imperative that management aimed to change of beliefs and behaviours, in tandem with pain which the IAYT helped these patients with. Another important aspect of IAYT is its holistic approach of mind -body component which has helped improve their quality of thoughts. With its daily practices the patients were able to overcome their earlier fear for their recovery. There was positive outlook and enthusiasm to participate in their daily life activities with much vigour and zest for life. Case 1 kept mentioning about how she felt physically lighter and mentally fresh after her morning practice. In fact the day she would skip her practice due to inevitable situation, she would feel as if she had missed something significant. Both the patients attributed IAYT for inner satisfaction and peace within, and sustainable decrease of irritability that they had earlier felt.

The Yoga components involve mindfulness, engaging postures correctly, awareness of breathing, relaxation and focused attention to sensation which are reputed to be of utmost important to obtain full benefits of the practice, rather than just quantifying the duration of practice (16). The patients were able to incorporate these important features from day one in their practice which led to better awareness of their system not only while they were practising. But also, in their daily life, they were able to integrate this and be mindful of their life as a whole. Along with their other personal improvements, their social behaviour also saw a huge change.

The economic impact related to low back pain is similar to other high-cost conditions, such as cardiovascular disease, cancer, mental health, and autoimmune diseases (17). This was noted in both the patients, where in case 1 it was her productivity loss in managing household work. In case of case 2, the patient had to leave his job since 5 months. In addition, the economic burden incurred for various other previous conventional interventions in private hospitals, time spent and also the travel expenses has reduced. In case of CLBP, interventions that have potential for cost effectiveness, safe, and feasible with minimum side effects having widespread accessibility is the need of the hour (9). In the present study, IAYT regime as an additional management was able to help the patients in overcoming the above mentioned burden points. Case 2 patient managed to resume his work eventually within the span of 1 month IAYT intervention.

Yoga as an adjunct treatment has emerged as a potential non-pharmacologic intervention, supported by various metaanalyses $(16,18,19)$, systematic review (20), clinical guidelines (13) and randomized controlled trials $(6,9,21-24)$ for CLBP. Also, given the low number of adverse events from previous studies, as well as our present cases, Yoga has proved itself again as a recommended additional therapy to patients. 


\section{Conclusion}

The present case study strengthens the possibilities of IAYT for being a cost effective adjunct intervention which might prove to be useful for clinical translation and integration.

\section{Acknowledgement}

The authors acknowledge the efforts of the patients for their availability to participate and discuss during the compilation of the data. Special thanks to Dr. Yashpal, Professor and Head of the Department of Cardiology at PGIMER, Chandigarh for his cooperation and support to conduct the IAYT sessions at Yogashala of the Cardiac Centre. Futher facilitated by MOU between PGIMER and SVYASA.

\section{Authorship contribution}

SP: Manuscript preparation, Delivery of the Yoga Protocol, Data Collection, Analysis and Interpretation of Data.

KM: Formulation of the Yoga Protocol

SJG: Manuscript preparation, Data Collection

PV: Delivery of the Yoga Protocol, Data collection

BG: Conceptualization, Design of the study, Supervision of the Yoga Protocol, Data Collection, Manuscript editing, reviewing and approval.

\section{Informed consent}

Yes, the written informed consent has been obtained from the patients. Necessary precautions were taken to respect the privacy and the confidentiality of the patient's information.

\section{Conflict of interest}

The Authors declare no conflict of interest.

Received Date: 26-01-20; Revised Date: 10-02-20

Accepted Date: $11-02-20$

\section{Source of funding}

None declared

\section{References}

1. Buchbinder R, van Tulder M, Öberg B, Costa LM, Woolf A, Schoene M, et al. Low back pain: a call for action. Lancet. 2018;391:2384-8.

2. Vos T, Allen C, Arora M, Barber RM, Brown A, Carter A, et al. GBD 2015, Global, regional, and national incidence, prevalence, and years lived with disability for 310 diseases and injuries, 1990-2015: a systematic analysis for the Global Burden of Disease Study 2015. Lancet. 2016;388:1545-602.

3. James SL, Abate D, Abate KH, Abay SM, Abbafati C, Abbasi N, et al. GBD 2017, Global, regional, and national incidence, prevalence, and years lived with disability for 354 Diseases and Injuries for 195 countries and territories, 1990-2017: A systematic analysis for the Global Burden of Disease Study 2017. Lancet. 2018;1789-858.

4. Ahdhi G, Subramanian R, Saya G, Yamuna T. Prevalence of low back pain and its relation to quality of life and disability among women in rural area of Puducherry, India. Indian J Pain. 2016;30:111.

5. Day MA, Ward LC, Ehde DM, Thorn BE, Burns J, Barnier A, et al. A Pilot Randomized Controlled Trial Comparing Mindfulness Meditation,
Cognitive Therapy, and Mindfulness-Based Cognitive Therapy for Chronic Low Back Pain. Pain Med. 2019;00:1-15.

6. Patil N, Nagaratna R, Tekur P, Manohar P, Bhargav H, Patil D. A randomized trial comparing effect of yoga and exercises on quality of life in among nursing population with chronic low back pain. Int J Yoga. 2018;11:208.

7. Patil NJ, Nagarathna R, Tekur P, Patil DN, Nagendra HR, Subramanya P. First phase: Designing of integrated yoga therapy module for chronic low back pain Second phase: Validation of integrated yoga therapy module for chronic low back pain Third phase: Feasibility study Results: Feasibility study. Int J Yoga. 2015;26-8.

8. Tekur P, Chametcha S, Hongasandra R, Raghuram N. Effect of yoga on quality of life of CLBP patients: A randomized control study. Int J Yoga. 2010;3:10.

9. Cherkin DC, Sherman KJ, Balderson BH, Cook AJ, Anderson ML, Hawkes RJ, et al. Effect of mindfulness-based stress reduction vs cognitive behavioral therapy or usual care on back pain and functional limitations in adults with chronic low back pain: A randomized clinical trial. JAMA J Am Med Assoc. 2016;315:1240-9.

10. Goyal M, Haythornthwaite JA. Is It Time to Make Mind-Body Approaches Available for Chronic Low Back Pain? Data Sharing An Ethical and Scientific Imperative. JAMA - J Am Med Assoc. 2016;315:1236-7.

11. Foster NE, Anema JR, Cherkin D, Chou R, Cohen SP, Gross DP, et al. Prevention and treatment of low back pain: evidence, challenges, and promising directions. Lancet. 2018;391:2368-83.

12. Steffens D, Maher CG, Pereira LSM, Stevens ML, Oliveira VC, Chapple M, et al. Prevention of lowback pain a systematic review and metaAnalysis. JAMA Intern Med. 2016;176:199-208.

13. Qaseem A, Wilt TJ, McLean RM, Forciea MA. Noninvasive treatments for acute, subacute, and chronic low back pain: A clinical practice guideline from the American College of Physicians. Ann Intern Med. 2017; 166:514-30

14. Chang DG, Holt JA, Sklar M, Groessl EJ, Diego S, Diego S, et al. U. S. Department of Veterans Affairs review of the literature. J Orthop Rheumatol. 2016;3:1-8

15. Hartvigsen J, Hancock MJ, Kongsted A, Louw Q Ferreira ML, Genevay S, et al. What low back pain is and why we need to pay attention. Lancet. 2018;391:2356-67.

16. Holtzman S, Beggs RT. Yoga for chronic low back pain: a meta-analysis of randomized controlled trials. Pain Res Manag. 2013;18:267-72.

17. Andrasinova T, Kalikova E, Kopacik R, Srotova I, Vlckova E, Dusek L, et al. Evaluation of the Neuropathic Component of Chronic Low Back Pain. Clin J Pain. 2019;35:7-17.

18. Holger Cramer, MSc, Romy Lauche, PhD, Heidemarie Haller, MSc, and Gustav Dobos M, Objectives: A Systematic Review and Meta-analysis of Yoga for. Clin J Pain. 2013;00:1-11.

19. Büssing A, Ostermann T, Lüdtke R, Michalsen A. Effects of yoga interventions on pain and pain-associated disability: A meta-analysis. J Pain. 2012;13:1-9.

20. Wieland L, Skoetz N, Pilkington K, Vempati R, D Adamo C, Berman B. Yoga treatment for chronic non-specific low back pain. Cochrane Database Syst Rev. 2017;1:CD010671

21. Saper RB, Lemaster C, Delitto A, Sherman KJ, Herman PM, Sadikova E, et al. Yoga, physical therapy, or education for chronic low back pain: A randomized noninferiority trial. Ann Intern Med. 2017;167:85-94.

22. Tilbrook HE. Yoga for Chronic Low Back Pain. Ann Intern Med. 2011;155:569-78

23. Sherman KJ, Cherkin DC, Wellman RD, Cook AJ, Hawkes RJ, Delaney K, et al. A randomized trial comparing yoga, stretching, and a self-care book for chronic low back pain. JAMA- Arch Intern Med. 2011;171: 2019-26.

24. Groessl EJ, Liu L, Chang DG, Wetherell JL, Bormann JE, Atkinson JH, et al. Yoga for Military Veterans with Chronic Low Back Pain: A Randomized Clinical Trial. Am J Prev Med [Internet]. 2017;53:599-608. 